\title{
RNA imaging in situ
}

\author{
Monya Baker \\ Rapidly maturing techniques reveal messengers within cells.
}

With RNA, as with many things, seeing can bring understanding. The presence of RNA in particular cells and sites within those cells can reveal mechanisms behind differentiation. In cancer and other diseases, RNA transcripts are sometimes found in the wrong places. "That's why everyone is interested in RNA localization, and the challenge has been to image the RNA," explains Samie Jaffrey, who studies RNA regulation at Weill Cornell Medical College.

In the last 12 months, the ability to visualize RNA has settled a half-century-long debate about how influenza virus packages its genes ${ }^{1}$, helped reveal how deeply H1N1 RNA penetrates lung tissue ${ }^{2}$, uncovered surprising dynamics of splicing ${ }^{3}$, shown that promoters on RNA transcripts regulate the transcripts' stability ${ }^{4}$, and demonstrated that neuronal mRNA is trafficked into dendrites as single transcripts on individual granules ${ }^{5}$. Imaging may be particularly important for regulatory RNAs that are otherwise hard to study, says Jaffrey. "Understanding the location of noncoding RNA might help us understand their function."

Advances in biochemistry, imaging and labeling are expanding the ways researchers can look at RNA in individual cells. Reliable, convenient reagents are now commercially available to label RNA in fixed cells in a variety of contexts. Techniques to study RNA transcripts in living cells are more challenging, but they are also expanding and improving.

\section{RNA FISH finds single molecules in fixed cells}

In situ hybridization reveals RNA by using transcript-specific probes consisting of complementary oligonucleotides attached to fluorescent dyes or other markers. When RNA fluorescence in situ hybridization (FISH) was first reported in the 1980s, it was difficult to perform and had limited applications. But what was once an obscure art has become an accessible technique.

If certain vendors' hopes are realized, imaging RNA transcripts within cells will become as routine as immunostaining for protein. RNA labeling is possible where antibody-based protein labeling is problematic, says Yuling Luo, CEO of Advanced Cell Diagnostics, a clinical diagnostic company that also sells RNA-detecting probes for research. Suitable antibodies may not be available, a protein's structure or location may thwart labeling attempts, or the conditions for labeling one protein may be inappropriate for colabeling others, he explains. In contrast, RNA transcripts for vastly different proteins can all be detected under the

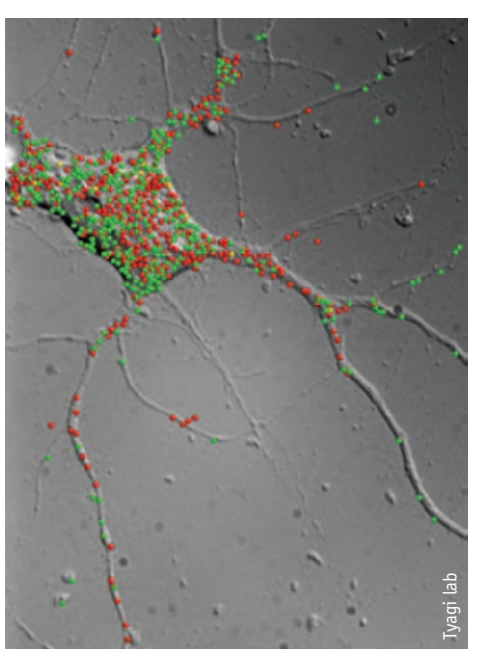

mRNAs for two genes were probed with single-molecule FISH probes in hippocampal neurons (here overlaid on a diffraction contrast image of the neuron). Red, MAP2; green, $\beta$-actin; yellow indicates when mRNAs were within 250 nanometers. whether a transcript is truly knocked down in RNA interference studies.

A huge driver for RNA FISH studies is a growing appreciation that cells encompass surprising heterogeneity, says Alexander van Oudenaarden, a systems biologist at the Massachusetts Institute of Technology and Hubrecht Institute. "If you ignore the individuality of the cells and combine them in one tube, you end up with an average individual that actually doesn't exist." He offers a toothsome metaphor: no one could learn the distinct flavors of mangoes, bananas or strawberries just by tasting fruit smoothies. Different cells can be right next to each other, he says, and detecting them requires single-cell resolution of gene expression.

van Oudenaarden has used RNA FISH on frozen slices of mouse intestine to show how same assay conditions.

Luo and others expect increasing use of RNA transcripts as biomarkers.

Gene expression profiling studies also raise questions RNA imaging can answer. Once scientists have homed in on a few transcripts for further study, RNA in situ hybridization can show how a gene is expressed across a population of cells, which pairs of transcripts rise and fall together within the same cell, and whether a transcript is localized in particular cell types or within special compartments like dendrites. In situ hybridization can also confirm transcripts for three types of stem cell markers are distributed within crypts, the invaginations between villi ${ }^{6}$. Intestinal crypts are often used to study regeneration and differentiation. With RNA in situ, one can quickly pick many markers and look at them in combinations to see which ones uniquely identify different cell types; doing the same with fluorescent protein labels would be impossible or impractical, he says. "You can make a lot of progress without having to genetically modify anything," says van Oudenaarden. "It's pretty easy. We joke that 

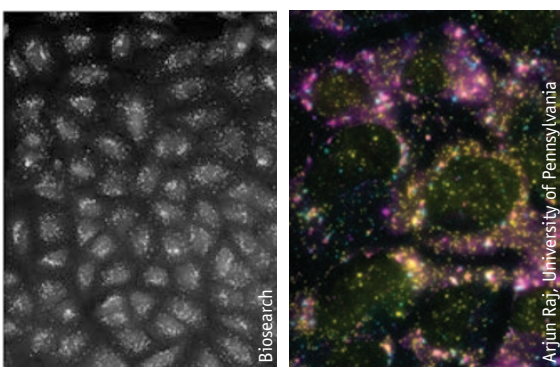

(Left) Stellaris FISH probes bound to mRNA transcripts encoding the transferrin receptor, imaged with an oil-free proprietary technology. (Right) Stellaris FISH RNA probes (yellow) and two immunostained proteins (magenta, cyan), imaged with a fluorescence microscope.

we are not even biochemists." What's difficult, he says, is designing imaging systems and accompanying software.

The first RNA FISH probes generally used plasmids to make antisense RNA, which incorporated modified nucleotides that bound to antibodies that could then be labeled. Robert Singer's lab, at Albert Einstein College of Medicine, created an alternative relying on long double-stranded DNA molecules with fluorescent nucleotide analogs incorporated enzymatically. They later improved the technique to use sets of smaller synthetic DNA probes, each about 50 nucleotides long and labeled with a handful of small-molecule fluorophores ${ }^{7}$.

The approach was further pared down by van Oudenaarden's former postdoc, Arjun Raj, who designed even shorter oligos for RNA FISH as a graduate student with Sanjay Tyagi at the University of Medicine and Dentistry of New Jersey ${ }^{8}$. This system uses sets of 50 or so probes, each targeting a different part of the transcript. These probes, each about 20 nucleotides long and modified with a single fluorophore, penetrate cells more easily and can be made more cheaply and quickly

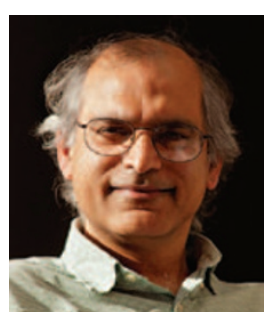

The ability to image RNAs is revealing new biomarkers and underappreciated heterogeneity across cells, says Tyagi.

manageable, says Tyagi. "It's a simple step that solves a big problem. Since the oligo synthesis has become automated, you can make oligos in a 96-well format." Greater numbers of smaller probes offer other advantages, he adds. For example, the binding of one probe relaxes transcripts so that other probes can bind more easily. And not every probe needs to be perfect. "You get a signal when all or most of them bind, but not when one or two bind by mistake."

Research tools company Biosearch Technologies launched its offering of short, singly labeled probes as Stellaris FISH in September last year. After researchers enter the sequence for the transcript they want online, the company software designs sets of up to 48 probes to label it. Biosearch offers a choice of eight fluorophores, ranging from blue to far red, but researchers can also buy unlabeled derivatized probes and add fluorophores themselves. van Oudenaarden, for example, attaches special fluorophores in the red end of the spectrum that can be imaged alongside blue-stained nuclei and green-labeled proteins. Tyagi uses two different fluorophores within the same set of probes to detect RNA processing events, such as alternate splice forms. A similar approach can identify fusion genes.

The standard pricing is $\$ 575$ for 5 nanomoles of pooled custom-made oligos, enough for 500-2,000 reactions, says Marc Beal, Director of Corporate Development at Biosearch Technologies. The system works with a wide variety of fluorescence microscopes, including wide field and confocal, and in fixed, frozen and paraf-

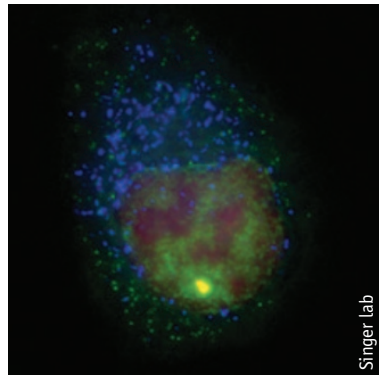

The central dogma in color: transcription (bright green nucleus), RNA moving through nuclear pores, single mRNAs in the cytoplasm (green dots) and the protein made from those mRNAs (blue). fin-embedded tissue samples as well as in cultured cells, says Beal. The 'sweet spot' is 60 - to 100 -fold magnification with an oilimmersed lens, though some customers have been able to see RNA spots using lower magnification. Biosearch is also working on a proprietary imaging system that will automate analysis.

Affymetrix and Advanced Cell Diagnostics separately offer other RNA probes initially developed at the reagents company Panomics. Instead of 48 probes that each bind the transcript of interest, these products use a series of four types of probes along with branching DNA technology to amplify the signal from a single RNA transcript.

First, two probes, each 25 bases long, bind to nearby spots on the same transcript. This creates a landing platform for another set of probes and eventually provides binding sites for labeled probes, with as many as 8,000 fluorophores attached to a single transcript. Signal detection occurs "only when two adjacent but independent hybridization events take place, a process that significantly reduces background of the assay," says George Bers, business manager of gene expression at Affymetrix. "The beauty of this system is that each RNA molecule appears as a single dot and you can quantify based on counting the dots," says Luo, who is collaborating with imaging software companies to create analysis tools that will count dots automatically.

As many as four transcripts can be detected at once, and the technique works in cultured cells, in fresh, frozen and paraffin-embedded tissue samples, and in circulating blood cells and fineneedle aspirates. Researchers can choose from ready-made probes, have custom probes made or have assays performed as a service. In addition to FISH, both Affymetrix and Advanced Cell Diagnostics also offer chromogenic stains detectable with brightfield microscopes, which show cell morphology more clearly and are used more often by clinical pathologists than fluorescence microscopes. Affymetrix probe sets can be ordered in multiple sizes and used for several assay types. For \$341, for example, researchers can purchase 440 microliters, enough for 44 assays in tissue samples or 730 cell assays in 96-well plates.

The vendors are developing or already offer systems for automatic slide preparation and analysis. They also report that many of their customers are trying RNA in situ hybridization for the first time, often to follow up on transcripts identified in broader gene expression studies. Results are more intuitive than graphs of gene expression, says Beal. "People can see what they've experimented on." 
As with any technology, researchers need appropriate controls, says Tyagi. All the vendors offer kits that detect a transcript of interest alongside that for another gene as a positive control. If possible, scientists should also test cells lacking the transcript to check for off-target labeling. In one study involving 15 transcripts, 13 worked straight out of the box, says van Oudenaarden. For the other two, problems were readily resolved.

\section{Engineering transcripts for live-cell imaging}

No matter how easy and accurate it is, imaging RNA in fixed cells can reveal only limited information. "We know the end state, but we don't know what it looks like when it's getting there," says Liz Gavis, a molecular biologist at Princeton.

There are options. For one, researchers can inject fluorescently labeled RNAs into cytoplasm. As an alternative, Sanjay Tyagi has developed a class of probes called molecular beacons. These are oligonucleotides with a fluorophore on one end and a quencher on the other. On its own, the probe folds up on itself, keeping the two ends in close contact and the beacon dark. Once hybridized to its target, the beacon unfurls and relays its signal. In practice, using molecular beacons often requires engineering transcripts to contain multiple binding sites. For both fluorescently labeled mRNAs and molecular beacons, introducing oligos is difficult, can damage cells and bypasses events that occur in the nucleus.

One solution is to genetically encode systems that label freshly transcribed genes as

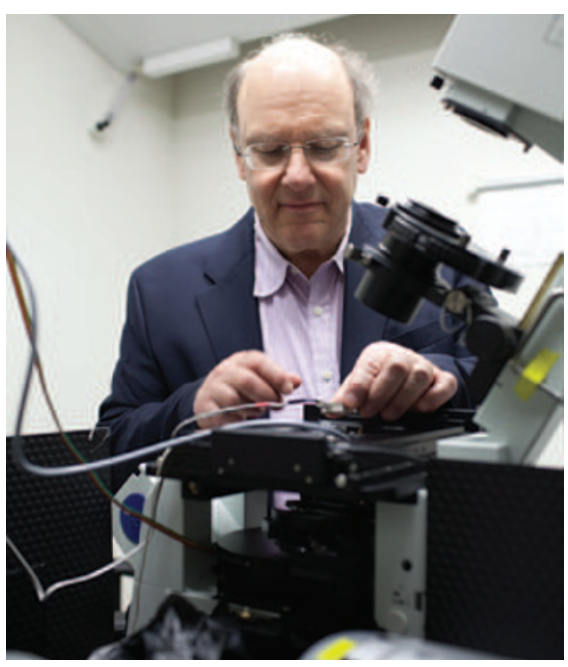

RNA imaging techniques can be made to work reliably in live and fixed cells, says Singer. soon as they are made in the nucleus. "To understand how RNA moves around in cells, we needed a way to look at RNA in living cells," says Singer, who developed the first and most popular method for coating RNA transcripts with fluorescent proteins ${ }^{9}$. Singer's system uses two elements from a bacteriophage called MS2. The transcript to be studied is modified with a series of MS2 RNA sequences that fold into loops. These 19-nucleotide structures are very tightly bound by the MS2 capsid protein, which is fused to a fluorescent reporter. Similar

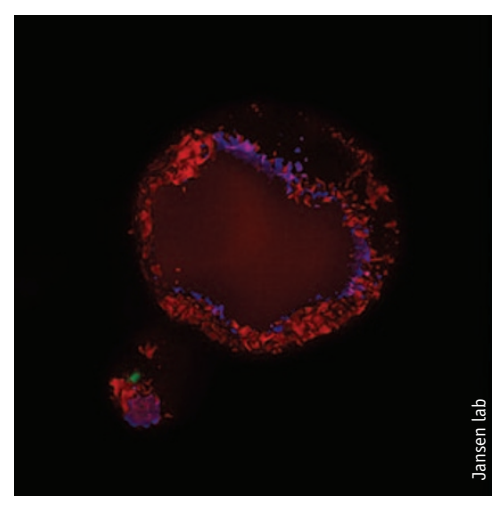

This pseudo three-dimensional picture demonstrates the spatial proximity of a localized mRNA (WSC2, green) and endoplasmic reticulum (red and purple). eggs, uses wide-field deconvolution microscopy, which requires additional software to distinguish signal from noise ${ }^{11}$.

Ralf Jansen at the University of Tübingen has used these reporter systems to study how mRNAs accumulate at appropriate organelles such as mitochondria and peroxisomes. His lab can detect single transcripts in yeast and human cancer cell lines. Doing so requires engineering more loops into transcripts so that they capture more fluorescent reporters: a total of 12 loops for yeast and 24 for humans. Systems such as MS2 systems, such as boxB and PP7, have been developed with other phage proteins and can be used together to label different transcripts in the same cell.

Such systems have been reported in bacteria, yeast, amoebae, fruit flies and mammalian cells. Recently, Singer and colleagues created a transgenic mouse carrying 24 MS2 binding sites in the essential $\beta$-actin gene in its native chromosome location and performed live-cell imaging in fibroblasts and primary neurons ${ }^{10}$. Though $\beta$-actin is a particularly abundant transcript, Singer anticipates that the approach will work for many genes. In the future, mice expressing MS2-engineered transcripts can be bred with a variety of mice expressing a range of MS2 fluorescent reporters, says Singer, thus greatly expanding what can be studied in mammalian systems. Until now, he says, "we haven't been able to investigate RNA and how it behaves in living intact tissues. Everything that we know is a result of cultured cells."

Different model systems pose different challenges. Imaging a three-dimensional, yolk-filled fruit fly egg and imaging cultured cells require different techniques, explains Gavis, who works with fly embryos and oocytes. Gavis uses a confocal microscope with a high capture rate because the RNA transcripts she studies move at speeds around a micron per second. Her lab has also adapted particle-tracking software, which can follow RNAs moving in all directions. Daniel St. Johnston at the Gurdon Institute, who has also adapted MS2 to fly are relatively easy to set up, he says, but scientists must remember that they are working with an artificial system. "You change the length of the RNA and the structure of the RNA, and you have to be very careful that that doesn't change the fate of the RNA." For example, most labs perform RNA FISH to make sure that engineered transcripts are distributed as expected.

In Jansen's experience, adding binding sites to transcripts rarely affects how the RNA is localized, but it may destabilize transcripts and reduce their abundance. Effects are hard to predict and must be verified by transcript. Jansen's lab checks decay rates using quantitative PCR and northern blots. (If an engineered transcript is unstable, cutting down on the number of stem loops can help, says Jansen, and so can switching to a system with smaller stem loops.) Also, engineered constructs must be re-evaluated for every cell type, as native RNA binding proteins vary by species and tissue, he says. When possible, Gavis recommends knocking out the endogenous transcript to check that engineered transcripts restore the phenotype, thereby indicating that they have been processed and translated appropriately.

\section{Emerging methods for live-cell RNA imaging}

A way to label unmodified, native transcripts has recently been described by Takeaki Ozawa at the University of Tokyo and colleagues. Instead of using fluorescently labeled phage proteins, 
transcripts are labeled with Pumilio, a human RNA binding protein that, unlike most such proteins, recognizes the sequence of an RNA transcript rather than its secondary structure. Ozawa engineered two versions of Pumilio to recognize adjacent 8 -nucleotide stretches on endogenous, unmodified transcripts of the $\beta$-actin gene. Each version also carries different halves of green fluorescent protein (GFP). When both versions of Pumilio bind the same transcript, they bring the two halves together,

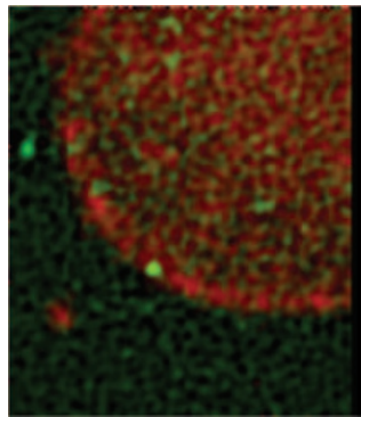

An mRNA moving through a nuclear pore shows up as a bright spot at the nuclear edge. The image uses a technique called crossregistration ${ }^{15}$.
Fluorescent Timer, which shifts from green to red fluorescence, could be useful for imaging transcripts at different time points.

A p t a m e r s, R N A sequences designed to bind other molecules, represent another exciting possibility. Jaffrey has engineered aptamers that become fluorescent after capturing a chromophore based on the one found in green fluorescent protein. When one of these engineered sequences, a sequence dubbed Spinach, was appended to a small RNA associated with the ribosome, researchers restoring fluorescence. Using a total-internal-reflection fluorescence microscope, Ozawa and colleagues were able to see individual, singly labeled $\beta$-actin transcripts and estimate how quickly mRNAs move along microtubules $^{12}$. Ozawa has also created versions of Pumilio that carry full-length GFP. Though brighter, they have higher background ${ }^{13}$. For both systems, researchers need appropriate equipment to see singly labeled transcripts. As with MS2, they also need to perform control experiments to ensure that attached Pumilio does not alter RNA transport or processing.

Meanwhile, Ozawa is looking to engineer proteins that recognize more transcripts, and he is also experimenting with other reporters. In particular, the protein could watch the RNAs leave the nucleus and distribute themselves throughout the cytosol in the expected patterns ${ }^{14}$. Because neither transcript nor chromophore fluoresces when unbound, background is minimal. What's more, the chromophore-bound aptamer is only a fraction of the size of an RNA binding protein fused to GFP.

A single-copy Spinach is not currently bright enough to be used for tracking mRNA, which is less abundant in cells than ribosomal RNA. However, Jaffrey sees much potential for improvement. To make transcripts brighter, he is appending multiple copies of the 80 -nucleotide aptamer sequence to the same transcript. Meanwhile, Jaffrey is creating more options, dubbed Radish and Carrot, in the reddish colors, shades that are more distinct from cells' natural autofluorescence. Jaffrey decided to name new colors after vegetables in homage to researchers who developed a rainbow of fluorescent proteins and often named them after fruits. The names also augur well for an upcoming smorgasbord of techniques to see RNAs in action.

Corrected after print 10 August 2012.

1. Chou, Y.Y. et al. Proc. Natl. Acad. Sci. USA 109, 9101-9106 (2012).

2. Everitt, A.R. et al. Nature 484, 519-523 (2012)

3. Vargas, D.Y. et al. Cell 147, 1054-1065 (2011).

4. Trcek, T. et al. Cell 147, 1484-1497 (2011).

5. Batish, M., van den Bogaard, P., Kramer, F.R. \& Tyagi, S. Proc. Natl. Acad. Sci. USA 109, 46454650 (2012).

6. Itzkovitz, S. et al. Nat. Cell Biol. 14, 106-114 (2012).

7. Femino, A.M., Fay, F.S., Fogarty, K. \& Singer, R.H. Science 280, 585-590 (1998).

8. Raj, A., van den Bogaard, P., Rifkin, S.A., van Oudenaarden, A. \& Tyagi, S. Nat. Methods 5 877-879 (2008).

9. Bertrand, E. et al. Mol. Cell 2, 437-445 (1998).

10. Lionnet, T. et al. Nat. Methods 8, 165-170 (2011).

11. Belaya, K. \& St Johnston, D. Methods Mol. Biol. 714, 265-283 (2011).

12. Yamada, T., Yoshimura, H., Inaguma, A. \& Ozawa, T. Anal. Chem. 83, 5708-5714 (2011).

13. Yoshimura, H., Inaguma, A., Yamada, T. \& Ozawa, T. ACS Chem. Biol. 7, 999-1005 (2012).

14. Paige, J.S., Wu, K.Y. \& Jaffrey, S.R. Science 333 , 642-646 (2011).

15. Grünwald, D. \& Singer, R. Nature 467, 604-607 (2010).

Monya Baker is technology editor for

Nature and Nature Methods

(m.baker@us.nature.com). 


\section{Erratum: RNA imaging in situ}

Monya Baker

Nat. Methods 9, 787-790 (2012); published online 30 July 2012; corrected after print 10 August 2012.

In the version of this article initially published, the image on page 787 should have been credited to the Tyagi lab. The error has been corrected in the PDF and HTML versions of this article. 